\title{
Two-Stage Procedures for the Bounded Risk Point Estimation of the Parameter and Hazard Rate of two Families of Distributions
}

\author{
Nitis Mukhopadhyay*, Ajit Chaturvedi ${ }^{\dagger}$, Ananya Malhotra ${ }^{\dagger}$
}

September 14, 2017

\begin{abstract}
Two families of distributions are considered which cover many distributions as specific cases. The problem of bounded risk point estimation of the parameter and hazard rate function of the two families of distribution is handled. Motivated by Mukhopadhyay and Pepe (2006), Roughani and Mahmoudi (2015) and Mahmoudi and Lalehzari (2017), two-stage procedures are developed based on maximum likelihood estimator (MLE) as well as uniformly minimum variance unbiased estimator (UMVUE). The estimation problem based on minimum mean square estimator (MMSE) is also considered. We establish that MMSE of the parameter and hazard rate provide a smaller risk.

Keywords and Phrases: Bounded risk estimation; Exact distributions; Moore and Bilikam family of distributions; Hazard rate; Sequential estimation; Stopping variables; Two-stage sampling.
\end{abstract}

\section{Introduction and the Fixed Sample Size Results}

Sequential estimation procedures are adopted in cases where the total sample size is not a degenerate random variable. Instead, data is evaluated as it is collected and further sampling is ceased in accordance with a predefined stopping rule as soon as significant results are achieved. Sequential

${ }^{*}$ Department of Statistics, University of Connecticut, Storrs, USA

${ }^{\dagger}$ Department of Statistics, University of Delhi, Delhi 110007, India 
procedures are also adopted in cases where no estimation procedures employing a non-random sample size can obtain the desired goals. For instance, one cannot achieve a minimum risk of an estimator where the sample size is fixed. Thus, in sequential analysis we may be able to arrive at a decision at a much earlier stage and consequently lower financial and/or human cost. Bounded risk estimation is a common sequential analysis problem consisting of a pre-assigned accuracy. Populations with known variance have a fixed sample size solution and no sequential methods need to be adopted. Sequential procedures come in handy when nothing is known about the population. Stein (1945) proposed a two-stage bounded risk estimation procedure for the normal distribution. He utilised the standard deviation of the initial sample to yield a terminal sample size. Later exponential and gamma distributions were considered to yield modelling time results because unlike normal distribution, modelling times are often skewed to the right.

A lot of work has been done in the literature of sequential estimation of the scale parameter of exponential distribution. Starr and Woodroofe (1972), Mukhopadhyay (1980, 1994), Isogai and Uno (1994), Mukhopadhyay and Datta (1995), Uno et al. (2004), Zacks and Mukhopadhyay (2006a,b), Zacks (2009) are some of the authors to name a few. Woodroofe (1977) developed sequential estimation of the scale parameter of gamma distribution. Takada and Nagata (1992) and Zacks and Khan (2011) studied the confidence intervals of the mean and scale parameter of gamma distribution. Mukhopadhyay and Pepe (2006) estimated the scale parameter of exponential distribution using its MLE/ UMVUE and two-stage procedures. Mahmoudi and Roughani (2015) developed a two-stage sampling scheme for estimating the scale parameter of gamma distribution by its MLE/ UMVUE assuming the shape parameter is known such that the risk is uniformly bounded by a pre-assigned number. They provided explicit formulas for the distribution and expected value of the stopping variable. In continuation to this study, Roughani and Mahmoudi (2015) provided explicit formulas for the expected value and risk of the MLE of the scale parameter in a two-stage sampling scheme. Later, Mahmoudi and Lalehzari (2017) considered a two-stage point estimation of the hazard rate of exponential distribution. One may be interested in the hazard function as they measure the conditional probability of failure given the system is currently working.

The purpose of the present paper is many-fold. First we consider a family of distributions by Chaturvedi and Alam (2010) which covers exponential and gamma distributions as specific cases. We generalise some of the results by Mukhopadhyay and Pepe (2006), Mahmoudi and Roughani (2015) and Roughani and Mahmoudi (2015) and also compare these results on the 
basis of two estimators, i.e. MMSE and MLE/ UMVUE of the unknown parameter. First we derive these estimators, i.e. MMSE, MLE and UMVUE of the parameter. Under Stein's two-stage sampling scheme, we derive explicit expressions of the stopping variable such that the risk of the estimators is uniformly bounded by a pre-assigned number. Next we derive the exact risk of both the estimators of the parameter and establish that the risk is smaller when we estimate the parameter by its MMSE. We now proceed by considering a family of distributions by Moore and Bilikam (1978) which covers exponential distribution as a specific case. We extend the results of Mahmoudi and Lalehzari (2017) and compare these results on the basis of two estimators, i.e. MMSE and MLE/ UMVUE of the hazard rate. First we derive these estimators, i.e. MMSE, MLE and UMVUE of the hazard function. Under Stein's two-stage sampling scheme, we derive explicit expressions of the stopping variable such that the risk of the estimators is uniformly bounded by a pre-assigned number. Next we derive the exact risk of both the estimators of the hazard rate and establish that the risk is smaller when we estimate the hazard rate by its MMSE.

Let $X$ be a random variable $(r v)$ from a family of distributions $\boldsymbol{F}_{\mathbf{1}}$ proposed by Chaturvedi and Alam (2010) with probability density function $(p d f)$

$$
f(x ; a, \alpha, \beta, \boldsymbol{\theta})=\frac{g^{\alpha-1}(x ; \boldsymbol{\theta}) g^{\prime}(x ; \boldsymbol{\theta})}{\beta^{\alpha} \Gamma(\alpha)} e^{-\frac{g(x ; \boldsymbol{\theta})}{\beta}} ; \quad x>a \geq 0, \alpha>0, \beta>0 .
$$

Here, $g(x ; \boldsymbol{\theta})$ is a function of $x$ and may also depend on a vector valued parameter $\boldsymbol{\theta}$ which is assumed to be known. Moreover, $g(x ; \boldsymbol{\theta})$ is a monotonically increasing function in $x$ with $g(a ; \boldsymbol{\theta})=0, g(\infty ; \boldsymbol{\theta})=\infty$ and $g^{\prime}(x ; \boldsymbol{\theta})$ denotes the derivative of $g(x ; \boldsymbol{\theta})$ with respect to $x, \alpha$ is known shape parameter while $\beta$ is unknown parameter. We note that $\boldsymbol{F}_{\mathbf{1}}$ covers the following distributions as specific cases:

I. For $g(x ; \boldsymbol{\theta})=x, \alpha=1$ and $a=0$, we get the one-parameter exponential distribution [Johnson and Kotz (1970, p.197)].

II. For $g(x ; \boldsymbol{\theta})=x$ and $a=0$, it gives gamma distribution. Further for integral values of $\alpha$, it gives Erlang distribution [Johnson and Kotz (1970, p.166)].

III. For $g(x ; \boldsymbol{\theta})=x^{p}, \boldsymbol{\theta}=p, p>0$ and $a=0$, it leads us to generalised gamma distribution [Johnson and Kotz (1970, p.197)].

IV. For $g(x ; \boldsymbol{\theta})=x^{p}, \boldsymbol{\theta}=p, p>0, \alpha=1$ and $a=0$, it turns out to be Weibull distribution [Johnson and Kotz (1970, p.250)]. 
V. For $g(x ; \boldsymbol{\theta})=x^{2}, \alpha=\frac{1}{2}$ and $a=0$, it is known as half-normal distribution [Davis (1952)].

VI. For $g(x ; \boldsymbol{\theta})=\frac{x^{2}}{2}, \alpha=\frac{m}{2}, m>0$ and $a=0$, we get Chi distribution [Patel et al. (1976, p.173)] and for $m=3$ we get Maxwell distribution [Tyagi and Bhattacharya (1989)].

VII. For $g(x ; \boldsymbol{\theta})=x^{2}, \alpha=1$ and $a=0$, it gives Rayleigh distribution [Sinha (1986, p.200)].

VIII. For $g(x ; \boldsymbol{\theta})=\log \left(1+x^{b}\right), \boldsymbol{\theta}=b, b>0, \alpha=1$ and $a=0$, it leads us to Burr distribution [Burr (1942), Cislak and Burr (1968)].

IX. For $g(x ; \boldsymbol{\theta})=\log \left(\frac{x}{a}\right), \alpha=1$ and $a>0$, it turns out to be Pareto distribution [Johnson and Kotz (1970, p.233)].

X. For $g(x ; \boldsymbol{\theta})=\log \left(1+\frac{x}{\nu}\right), \boldsymbol{\theta}=\nu, \nu>0, \alpha=1$ and $a=0$, it is called Lomax (1954) distribution.

XI. For $g(x ; \boldsymbol{\theta})=\log \left(1+\frac{x^{b}}{\nu}\right), \boldsymbol{\theta}=(\nu, b), \nu>0, b>0, \alpha=1$ and $a=0$, it becomes Burr distribution with scale parameter $\nu$ [Tadikamalla (1980)].

XII. For $g(x ; \boldsymbol{\theta})=\log \left(1+\frac{x^{b}}{\delta^{b}}\right), \boldsymbol{\theta}=(\delta, b), \delta>0, b>0, \alpha=1$ and $a=0$, it is called log-logistic distribution [Kleiber (2004)].

XIII. For $g(x ; \boldsymbol{\theta})=x^{2}, \alpha=k+1, k \geq 0$ and $a=0$, we get generalized Rayleigh distribution of Voda (1978).

XIV. For $g(x ; \boldsymbol{\theta})=x^{\gamma} e^{\nu x}, \boldsymbol{\theta}=(\gamma, \nu), \gamma>0, \nu>0, \alpha=1$ and $a=0$, it gives the modified Weibull distribution of Lai et al. (2003).

XV. For $\alpha=1$ and $a=0$, it leads us to the family of distributions considered by Gurvich et al. (1997).

XVI. For $g(x ; \boldsymbol{\theta})=\gamma \exp \left(\frac{x^{\nu}}{\gamma^{\nu}}-1\right), \boldsymbol{\theta}=(\gamma, \nu), \gamma>0, \nu>0, \alpha=1$ and $a=0$, it turns out to a modified form of Weibull distribution considered by Xie et al. (2002). If we take $\gamma=1$, it reduces to the lifetime distribution considered by Chen (2000).

XVII. For $g(x ; \boldsymbol{\theta})=(\gamma x)^{\nu}+(\mu x)^{\lambda}, \boldsymbol{\theta}=(\gamma, \nu, \mu, \lambda), \gamma>0, \nu>0, \mu>0, \lambda>0$, $\alpha=\beta=1$ and $a=0$, it becomes the additive Weibull distribution of Xie and Lai (1995) and Stoner et al. (1994). 
XVIII. For $g(x ; \boldsymbol{\theta})=\mu x+\nu \frac{x^{2}}{2}, \boldsymbol{\theta}=(\nu, \mu), \nu>0, \mu>0, \alpha=\beta=1$ and $a=0$, it is called linear exponential distribution.

XIX. For $g(x ; \boldsymbol{\theta})=(x-a)+\frac{\nu}{\gamma} \log \left(\frac{x+\nu}{a+\gamma}\right), \boldsymbol{\theta}=(\nu, \gamma), \nu>0, \gamma>0$ and $a=0$, we get the generalized Pareto distribution of Ljubo (1965).

XX. For $g(x ; \boldsymbol{\theta})=\alpha x^{2}, \boldsymbol{\theta}=\alpha$ and $a=0$, we get the Nakagami (1960) distribution.

Let $X_{1}, X_{2}, \ldots, X_{n}$ be a random sample from the family of distributions $\boldsymbol{F}_{\mathbf{1}}$. Then assuming $a, \alpha$ and $\boldsymbol{\theta}$ are known, the likelihood function of $\beta$ is given by

$$
\begin{aligned}
L(\beta \mid \mathbf{x}) & =\prod_{i=1}^{n} f\left(x_{i} ; a, \alpha, \beta, \boldsymbol{\theta}\right) \\
& =\frac{e^{-\frac{S_{n}}{\beta}}}{\beta^{n \alpha}} \prod_{i=1}^{n} \frac{g^{\alpha-1}\left(x_{i} ; \boldsymbol{\theta}\right) g^{\prime}\left(x_{i} ; \boldsymbol{\theta}\right)}{\Gamma(\alpha)},
\end{aligned}
$$

where $S_{n}=\sum_{i=1}^{n} g\left(x_{i} ; \boldsymbol{\theta}\right)$. Thus by factorisation theorem [see Rohtagi and Saleh (2012, p.361)], $S_{n}$ is sufficient statistic for $\beta$ and follows gamma distribution with shape parameter $n \alpha$ and scale parameter $\beta$. Since the distribution of $S_{n}$ belongs to the exponential family, it is also complete [see Rohtagi and Saleh (2012, p.367)]. For $q>0$, consider

$$
E\left(S_{n}^{q}\right)=\frac{\Gamma(n \alpha+q)}{\Gamma(n \alpha)} \beta^{q} .
$$

Thus the UMVUE of $\beta^{q}$ is

$$
\widetilde{\beta}_{U}^{q}=\frac{\Gamma(n \alpha)}{\Gamma(n \alpha+q)} S_{n}^{q}
$$

From 1.2 , the MLE of $\beta^{q}$ is

$$
\widetilde{\beta}_{M L}^{q}=\left(\frac{S_{n}}{n \alpha}\right)^{q} .
$$

For $q=1$, we see that the MLE is equal to the UMVUE of $\beta$ and let us denote them by

$$
\widetilde{\beta}_{n}=\frac{S_{n}}{n \alpha}
$$


based on sample size $n$. Now, for some number $k>0$, the MMSE of $\beta$ based on sample size $n$ can be obtained by minimising $E\left(\left(k S_{n}-\beta\right)^{2}\right)$ with respect to $k$ and we get

$$
\widehat{\beta}_{n}=\frac{S_{n}}{n \alpha+1} .
$$

Let us denote the loss function for estimating $\beta$ by its estimator $\widehat{\beta}_{n}$ by

$$
L\left(\widehat{\beta}_{n}, \beta\right)=A\left(\widehat{\beta}_{n}-\beta\right)^{2},
$$

where $A$ is a known positive weight. The associated risk is

$$
R\left(\widehat{\beta}_{n}, \beta\right)=E\left(A\left(\widehat{\beta}_{n}-\beta\right)^{2}\right)=\frac{A \beta^{2}}{n \alpha+1} .
$$

The loss of estimating $\beta$ by $\widetilde{\beta}_{n}$ is

$$
L\left(\widetilde{\beta}_{n}, \beta\right)=A\left(\widetilde{\beta}_{n}-\beta\right)^{2},
$$

and the associated risk is

$$
R\left(\widetilde{\beta}_{n}, \beta\right)=E\left(A\left(\widetilde{\beta}_{n}-\beta\right)^{2}\right)=\frac{A \beta^{2}}{n \alpha} .
$$

The sample sizes required to achieve $R\left(\widehat{\beta}_{n}, \beta\right) \leq \omega$ and $R\left(\widetilde{\beta}_{n}, \beta\right) \leq \omega$ are $n \geq n^{*}$ and $n \geq n^{* *}$ respectively, where

$$
n^{*}=\frac{1}{\alpha}\left(\frac{A \beta^{2}}{\omega}-1\right)
$$

and

$$
n^{* *}=\frac{A \beta^{2}}{\alpha \omega}
$$

We note that $R\left(\widehat{\beta}_{n}, \beta\right)<R\left(\widetilde{\beta}_{n}, \beta\right)$. Thus, if we consider a two-stage procedure motivated by MMSE, we expect a reduction in the risk. Since $n^{*}$ and $n^{* *}$ are unknown, there does not exist any fixed sample size procedure for this problem [see Takada (1986)].

Let $X$ be a random variable $(r v)$ from a family of distributions $\boldsymbol{F}_{\mathbf{2}}$ proposed by Moore and Bilikam (1978) with probability density function $(p d f)$

$$
f(x ; \nu, \theta)=\frac{\theta g^{\theta-1}(x) g^{\prime}(x)}{\nu} e^{-\frac{g^{\theta}(x)}{\nu}} ; \quad x \geq 0, \theta>0, \nu>0 .
$$


Here, $g(x)$ is a real-valued, strictly increasing function of $x$ with $g\left(0^{+}\right)=0$, $g(\infty)=\infty$ and $g^{\prime}(x)$ denotes the derivative of $g(x)$ with respect to $x, \theta$ is a known parameter while $\nu$ is an unknown parameter. We note that $\boldsymbol{F}_{\boldsymbol{2}}$ covers the following distributions as specific cases:

I. For $g(x)=x$ and $\theta=1$, we get exponential distribution [Johnson and Kotz (1970), p.166].

II. For $g(x)=x$, we obtain Weibull distribution [Johnson and Kotz (1970), p.250].

III. For $g(x)=\log \left(1+x^{b}\right), b>0$ and $\theta=1$, it gives Burr distribution [Burr (1942); Cislak and Burr (1968)].

IV. For $g(x)=\log \left(\frac{x}{a}\right), a>0$ and $\theta=1$, it leads us to Pareto distribution [Johnson and Kotz (1970), p.233].

V. For $g(x)=x$ and $\theta=2$, we obtain Rayleigh distribution ([Johnson and Kotz (1970), p.200].

VI. For $g(x)=\log \left(1+\frac{x}{\sigma}\right), \sigma>0$ and $\theta=1$, it is called Lomax (1954) distribution.

VII. For $g(x)=\log \left(1+\frac{x^{b}}{\sigma}\right), b>0, \sigma>0$ and $\theta=1$, it becomes Burr distribution with scale parameter $\sigma$ (Tadikamalla 1980).

VIII. For $g(x)=x^{\gamma} e^{\sigma x}, \gamma>0, \sigma>0$ and $\theta=1$, it gives modified Weibull distribution of Lai et al. (2003).

IX. For $g(x)=(x-a)+\frac{\sigma}{\lambda} \log \left(\frac{x+\sigma}{a+\lambda}\right), \sigma>0, \lambda>0, a \geq 0$ and $\theta=1$, we get generalised Pareto distribution of Ljubo (1965).

$\mathrm{X}$. For $g(x)=b x+\frac{\lambda}{2} x^{2}, \lambda>0, b>0$ and $\theta=1$, we get the linear exponential distribution [Mahmoud and Al-Nagar (2009)].

XI. For $g(x)=\left(1+x^{b}\right)^{\lambda}-1, b>0, \lambda>0$ and $\theta=1$, we get the generalised power Weibull distribution [Nikulin and Haghighi (2006)].

XII. For $g(x)=\frac{\alpha}{b}\left(e^{b x}-1\right), \alpha>0, b>0$ and $\theta=1$, we get the Gompertz distribution [Khan and Zia (2009)].

XIII. For $g(x)=\left(e^{x^{b}}-1\right), b>0$ and $\theta=1$, this gives Chen (2000) distribution. 
XIV. For $g(x)=(x-a), a \geq 0$ and $\theta=1$, we get the two-parameter exponential distribution (Ahsanullah (1980)).

Let $X_{1}, X_{2}, \ldots, X_{n^{\prime}}$ be a random sample from the Moore and Bilikam family of distributions $\boldsymbol{F}_{\mathbf{2}}$. Then assuming $\theta$ is known, the likelihood function of $\nu$ is given by

$$
\begin{aligned}
L(\nu \mid \mathbf{x}) & =\prod_{i=1}^{n^{\prime}} f\left(x_{i} ; \nu, \theta\right) \\
& =\left(\frac{\theta}{\nu}\right)^{n^{\prime}} e^{-\frac{S_{n^{\prime}}}{\nu}} \prod_{i=1}^{n^{\prime}} g^{\theta-1}\left(x_{i}\right) g^{\prime}\left(x_{i}\right)
\end{aligned}
$$

where $S_{n^{\prime}}=\sum_{i=1}^{n^{\prime}} g^{\theta}\left(x_{i}\right)$. Thus by factorisation theorem [see Rohtagi and Saleh (2012, p. 361)], $S_{n^{\prime}}$ is sufficient statistic for $\nu$ and follows gamma distribution with shape parameter $n$ and scale parameter $\nu$. Since the distribution of $S_{n^{\prime}}$ belongs to the exponential family, it is also complete [see Rohtagi and Saleh (2012, p.367)]. For $q>0$, consider

$$
E\left(S_{n^{\prime}}^{q}\right)=\frac{\Gamma\left(n^{\prime}+q\right)}{\Gamma\left(n^{\prime}\right)} \nu^{q}
$$

Thus the UMVUE of $\nu^{q}$ is $\widetilde{\nu}_{U}^{q}=\frac{\Gamma\left(n^{\prime}\right)}{\Gamma\left(n^{\prime}+q\right)} S_{n^{\prime}}^{q}$.

From (1.12), the MLE of $\nu^{q}$ is

$$
\widetilde{\nu}_{M L}^{q}=\left(\frac{S_{n^{\prime}}}{n^{\prime}}\right)^{q}
$$

For $q=1$, we see that the MLE is equal to the UMVUE of $\nu$ and let us denote them by

$$
\widetilde{\nu}_{n^{\prime}}=\frac{S_{n^{\prime}}}{n^{\prime}}
$$

based on sample size $n^{\prime}$. Now, for some number $k>0$, the MMSE of $\nu$ based on sample size $n^{\prime}$ can be obtained by minimising $E\left(\left(k S_{n^{\prime}}-\nu\right)^{2}\right)$ with respect to $k$ and we get

$$
\widehat{\nu}_{n^{\prime}}=\frac{S_{n^{\prime}}}{n^{\prime}+1}
$$

In similar fashion one can easily obtain the UMVUE, MLE and MMSE of $\frac{1}{\nu}$ as $\frac{n^{\prime}-1}{S_{n^{\prime}}}, \frac{n^{\prime}}{S_{n^{\prime}}}$ and $\frac{n^{\prime}-2}{S_{n^{\prime}}}$ respectively. 
The hazard rate at time point $t$ of the family of distributions $\boldsymbol{F}_{\mathbf{2}}$ is given by

$$
\gamma=\frac{\theta g^{\theta-1}(t) g^{\prime}(t)}{\nu}
$$

Thus, the MMSE of $\gamma$ based on sample size $n^{\prime}$ is

$$
\widehat{\gamma}_{n^{\prime}}=\frac{\theta g^{\theta-1}(t) g^{\prime}(t)\left(n^{\prime}-2\right)}{S_{n^{\prime}}} .
$$

The UMVUE of $\gamma$ based on sample size $n^{\prime}$ is

$$
\widetilde{\gamma}_{n^{\prime}-U}=\frac{\theta g^{\theta-1}(t) g^{\prime}(t)\left(n^{\prime}-1\right)}{S_{n^{\prime}}}
$$

and the MLE of $\gamma$ based on sample size $n^{\prime}$ is

$$
\widetilde{\gamma}_{n^{\prime}-M L}=\frac{\theta g^{\theta-1}(t) g^{\prime}(t) n^{\prime}}{S_{n^{\prime}}} .
$$

Let us denote the loss function for estimating $\gamma$ by $\widehat{\gamma}_{n^{\prime}}$ by

$$
L\left(\widehat{\gamma}_{n^{\prime}}, \gamma\right)=A^{\prime}\left(\widehat{\gamma}_{n^{\prime}}-\gamma\right)^{2}
$$

where $A^{\prime}$ is a known positive weight. The associated risk is

$$
R\left(\widehat{\gamma}_{n^{\prime}}, \gamma\right)=\frac{A^{\prime}\left(\theta g^{\theta-1}(t) g^{\prime}(t)\right)^{2}}{\left(n^{\prime}-1\right) \nu^{2}}
$$

The loss of estimating $\gamma$ by $\widetilde{\gamma}_{n^{\prime}-M L}$ is

$$
L\left(\widetilde{\gamma}_{n^{\prime}-M L}, \gamma\right)=A^{\prime}\left(\widetilde{\gamma}_{n^{\prime}-M L}-\gamma\right)^{2}
$$

and the associated risk is

$$
R\left(\widetilde{\gamma}_{n^{\prime}-M L}, \gamma\right)=A^{\prime} E\left(\left(\widetilde{\gamma}_{n^{\prime}-M L}-\gamma\right)^{2}\right)=\frac{A^{\prime}\left(\theta g^{\theta-1}(t) g^{\prime}(t)\right)^{2}\left(n^{\prime}+2\right)}{\left(n^{\prime}-1\right)\left(n^{\prime}-2\right) \nu^{2}}
$$

We see that the solution based on MLE of $\gamma$ for the bounded risk point estimation problem does not exist unless we apply Taylor's series expansion to get

$$
R\left(\widetilde{\gamma}_{n^{\prime}-M L}, \gamma\right)=A^{\prime} E\left(\left(\widetilde{\gamma}_{n^{\prime}-M L}-\gamma\right)^{2}\right) \approx \frac{A^{\prime}\left(\theta g^{\theta-1}(t) g^{\prime}(t)\right)^{2}}{n^{\prime} \nu^{2}}
$$


Now, the loss of estimating $\gamma$ by $\widetilde{\gamma}_{n^{\prime}-U}$ is

$$
L\left(\widetilde{\gamma}_{n^{\prime}-U}, \gamma\right)=A^{\prime}\left(\widetilde{\gamma}_{n^{\prime}-U}-\gamma\right)^{2}
$$

and the associated risk is

$$
R\left(\widetilde{\gamma}_{n^{\prime}-U}, \gamma\right)=A^{\prime} E\left(\left(\widetilde{\gamma}_{n^{\prime}-U}-\gamma\right)^{2}\right)=\frac{A^{\prime}\left(\theta g^{\theta-1}(t) g^{\prime}(t)\right)^{2}}{\left(n^{\prime}-2\right) \nu^{2}}
$$

Mahmoudi and Lalehzari (2017) have considered two-stage point estimation of the hazard rate of exponential distribution based on the MLE of its parameter and we have seen from (1.18) that the exact solution of risk of MLE does not exist. We also observe that the risk corresponding to UMVUE in (1.21) and MMSE in (1.16) are smaller than that of the MLE in (1.18). Another advantage of using UMVUE and MMSE is that one does not require Taylor's series expansion to get fixed sample size solution. The sample sizes required to achieve $R\left(\widehat{\gamma}_{n^{\prime}}, \gamma\right) \leq \omega^{\prime}$ and $R\left(\widetilde{\gamma}_{n^{\prime}-U}, \gamma\right) \leq \omega^{\prime}$ are $n^{\prime} \geq n^{\prime *}$ and $n^{\prime} \geq n^{\prime * *}$ respectively, where

$$
n^{\prime *}=\left(\frac{A^{\prime}\left(\theta g^{\theta-1}(t) g^{\prime}(t)\right)^{2}}{\omega^{\prime} \nu^{2}}+1\right)
$$

and

$$
n^{\prime * *}=\left(\frac{A^{\prime}\left(\theta g^{\theta-1}(t) g^{\prime}(t)\right)^{2}}{\omega^{\prime} \nu^{2}}+2\right) .
$$

We note that $R\left(\widehat{\gamma}_{n^{\prime}}, \gamma\right)<R\left(\widetilde{\gamma}_{n^{\prime}-U}, \gamma\right)$. Thus, if we consider a two-stage procedure motivated by MMSE, we expect a reduction in the risk. Since $n^{\prime *}$ and $n^{\prime * *}$ are unknown, there does not exist any fixed sample size procedure for this problem [see Takada (1986)].

The rest of the paper is organised as follows. In Section 2, we consider the family of distribution $\boldsymbol{F}_{\mathbf{1}}$. Then under Stein's two-stage sampling scheme, we determine the stopping variable such that the risk in estimating the parameter $\beta$ by its MMSE is uniformly bounded by $\omega$. Next we derive the exact risk in estimating $\beta$ by its MMSE. We also state the same results while estimating $\beta$ by its MLE/UMVUE and perform extensive numerical computations to compare the risks of MMSE and MLE/UMVUE of $\beta$. Finally in Section 3, we consider the family of distribution $\boldsymbol{F}_{\mathbf{2}}$ and under Stein's two-stage sampling scheme, we determine the stopping variable such that the risk in estimating the hazard rate $\gamma$ by its MMSE is uniformly bounded by $\omega^{\prime}$. Next we derive the exact risk in estimating $\gamma$ by its MMSE. We also state the same results while estimating $\gamma$ by its UMVUE and perform extensive numerical computations to compare the risks of MMSE and UMVUE of hazard rate $\gamma$. 


\section{Estimation of Parameter of $F_{1}$}

\subsection{Two-Stage Procedure based on Minimum Mean Square Estimator}

Let $X_{1}, X_{2}, \ldots, X_{m}$ be a pilot sample from the family of distributions $\boldsymbol{F}_{\mathbf{1}}$. Using Stein's two-stage sampling scheme [see Stein $(1945,1949)$ ], we propose the following stopping rule:

$$
N_{m}=N(m, B, \omega)=\max \left\{m,\left\lfloor\frac{1}{\alpha}\left(\frac{B \bar{S}_{m}^{2}}{\omega}-1\right)\right\rfloor+1\right\},
$$

where $\lfloor z\rfloor$ denotes the greatest integer less than $z . B$ is a positive coefficient and is determined such that the risk $R\left(\widehat{\beta}_{N_{m}}, \beta\right)$ is bounded by a pre-assigned number $\omega . \bar{S}_{m}=\frac{S_{m}}{m \alpha+1}=\frac{\sum_{i=1}^{m} g\left(x_{i}, \boldsymbol{\theta}\right)}{m \alpha+1}$. We shall later prove that $B$ is a function of all the known quantities $A, m$ and $\alpha$. Now, if $N_{m}=m$, then the pilot sample is large enough and we don't require to draw more observations at the second stage. But if $N_{m}>m$, then the pilot sample is not large enough and hence we must draw $N_{m}-m$ more observations at the second stage, say $X_{m+1}, X_{m+2}, \ldots, X_{N_{m}}$. Finally, based on all the observations from both the stages, $X_{1}, X_{2}, \ldots, X_{N_{m}}$, we estimate the parameter $\beta$ by its MMSE, i.e.

$$
\widehat{\beta}_{N_{m}}=\frac{S_{N_{m}}}{N_{m} \alpha+1}=\frac{\sum_{i=1}^{N_{m}} g\left(x_{i}, \boldsymbol{\theta}\right)}{N_{m} \alpha+1} .
$$

Thus the risk associated with this estimator is

$$
R\left(\widehat{\beta}_{N_{m}}, \beta\right)=E\left(A\left(\widehat{\beta}_{N_{m}}-\beta\right)^{2}\right) .
$$

Theorem 2.1. Consider the two-stage procedure in (2.1) and the loss function in (1.5) for sample size $N_{m}$. If we estimate $\beta$ by $\widehat{\beta}_{N_{m}}=\frac{S_{N_{m}}}{N_{m} \alpha+1}$, then for all fixed $\alpha, \beta, m$ and $A$, we conclude that $R\left(\widehat{\beta}_{N_{m}}, \beta\right) \leq \omega$ provided $m \alpha>2$ and

$$
B=B(m, A, \alpha)=\frac{A(m \alpha+1)^{2}(2 m \alpha+3)}{m \alpha(m \alpha-1)(m \alpha-2)} .
$$

The $r v N_{m}$ in (2.1) is a discrete $r v$ and can take values $\{m, m+1, \ldots\}$. We define

$$
\lambda_{j}=\left(\frac{m \alpha+1}{\beta}\right) \sqrt{\frac{\omega}{B}(j \alpha+1)}
$$


and $G(z ; a, b)$ as the cumulative distribution function $(c d f)$ of gamma distribution at the point $z$ with shape parameter $a$ and scale parameter $b$. Also let $\bar{G}(z ; a, b)=1-G(z ; a, b)$.

To derive the explicit expressions for the expected value and risk of $\widehat{\beta}_{N_{m}}$, let $\mathcal{F}_{m}=\sigma\left(X_{1}, X_{2}, \ldots, X_{m}\right)$ be the $\sigma$-field generated by the random variables at the first stage. Then we have

I. $\bar{S}_{m}=\frac{S_{m}}{m \alpha+1}$ is measurable in $\mathcal{F}_{m}$.

II. $N_{m}$ is measurable in $\mathcal{F}_{m}$.

III. $\bar{S}_{N_{m}-m}$ is independent of $\mathcal{F}_{m}$.

The following theorem provides the exact risk of MMSE of $\widehat{\beta}_{N_{m}}$.

Theorem 2.2. The risk of $\widehat{\beta}_{N_{m}}$ is

$$
\begin{aligned}
R\left(\widehat{\beta}_{N_{m}}, \beta\right)= & A \beta^{2}\left[1+\frac{m \alpha}{(m \alpha+1)} G\left(\lambda_{m} ; m \alpha+2,1\right)\right. \\
& +\sum_{n=m+1}^{\infty} \frac{m \alpha(m \alpha+1)}{(n \alpha+1)^{2}}\left\{\bar{G}\left(\lambda_{n-1} ; m \alpha+2,1\right)-\bar{G}\left(\lambda_{n} ; m \alpha+2,1\right)\right\} \\
& +\sum_{n=m+1}^{\infty} \frac{(n-m) \alpha}{(n \alpha+1)^{2}}\left\{\bar{G}\left(\lambda_{n-1} ; m \alpha, 1\right)-\bar{G}\left(\lambda_{n} ; m \alpha, 1\right)\right\} \\
& +\sum_{n=m+1}^{\infty}\left(\frac{(n-m) \alpha}{n \alpha+1}\right)^{2}\left\{\bar{G}\left(\lambda_{n-1} ; m \alpha, 1\right)-\bar{G}\left(\lambda_{n} ; m \alpha, 1\right)\right\} \\
& +\sum_{n=m+1}^{\infty} \frac{2 m(n-m) \alpha^{2}}{(n \alpha+1)^{2}}\left\{\bar{G}\left(\lambda_{n-1} ; m \alpha+1,1\right)-\bar{G}\left(\lambda_{n} ; m \alpha+1,1\right)\right\} \\
& -2 \frac{m \alpha}{m \alpha}+1^{\infty}\left(\lambda_{m} ; m \alpha+1,1\right) \\
& -\sum_{n=m+1}^{\infty} \frac{2 m \alpha}{n \alpha+1}\left\{\bar{G}\left(\lambda_{n-1} ; m \alpha+1,1\right)-\bar{G}\left(\lambda_{n} ; m \alpha+1,1\right)\right\} \\
& \left.-\sum_{n=m+1}^{\infty} \frac{2(n-m) \alpha}{n \alpha+1}\left\{\bar{G}\left(\lambda_{n-1} ; m \alpha, 1\right)-\bar{G}\left(\lambda_{n} ; m \alpha, 1\right)\right\}\right] .
\end{aligned}
$$

\subsection{Two-Stage Procedure based on Maximum Likeli- hood Estimator}

Let $X_{1}, X_{2}, \ldots, X_{m}$ be a pilot sample from the family of distributions $\boldsymbol{F}_{\mathbf{1}}$. Using Stein's two-stage sampling scheme [see Stein $(1945,1949)$ ], we propose 
the following stopping rule:

$$
N_{m}^{\prime}=N\left(m, B^{\prime}, \omega\right)=\max \left\{m,\left\lfloor\frac{B^{\prime} \bar{S}_{m}^{2}}{\alpha \omega}\right\rfloor+1\right\},
$$

where $B^{\prime}$ is a positive coefficient and is determined such that the risk $R\left(\widetilde{\beta}_{N_{m}^{\prime}}, \beta\right)$ is bounded by a pre-assigned number $\omega . \bar{S}_{m}=\frac{S_{m}}{m \alpha}=\frac{\sum_{i=1}^{m} g\left(x_{i}, \boldsymbol{\theta}\right)}{m \alpha}$. Based on all the observations from both the stages, $X_{1}, X_{2}, \ldots, X_{N_{m}^{\prime}}$, we estimate the parameter $\beta$ by its MLE/UMVUE, i.e.

$$
\widetilde{\beta}_{N_{m}^{\prime}}=\frac{S_{N_{m}^{\prime}}}{N_{m}^{\prime} \alpha}=\frac{\sum_{i=1}^{N_{m}^{\prime}} g\left(x_{i}, \boldsymbol{\theta}\right)}{N_{m}^{\prime} \alpha} .
$$

Thus the risk associated with this estimator is

$$
R\left(\widetilde{\beta}_{N_{m}^{\prime}}, \beta\right)=E\left(A\left(\widetilde{\beta}_{N_{m}^{\prime}}-\beta\right)^{2}\right) .
$$

Theorem 2.3. Consider the two-stage procedure in $(2.2)$ and the loss function in (1.7) for sample size $N_{m}^{\prime}$. If we estimate $\beta$ by $\widetilde{\beta}_{N_{m}^{\prime}}=\frac{S_{N_{m}^{\prime}}}{N_{m}^{\prime} \alpha}$, then for all fixed $\alpha, \beta, m$ and $A$, we conclude that $\left.R \widetilde{\beta}_{N_{m}^{\prime}}, \beta\right) \leq \omega$ provided $m \alpha>2$ and

$$
B^{\prime}=B^{\prime}(m, A, \alpha)=\frac{2 A m \alpha(m \alpha+1)}{(m \alpha-1)(m \alpha-2)} .
$$

The $r v N_{m}^{\prime}$ in 2.2 is a discrete $r v$ and can take values $\{m, m+1, \ldots\}$. We define

$$
\lambda_{j}^{\prime}=\left(\frac{m \alpha}{\beta}\right) \sqrt{\frac{\alpha \omega}{B^{\prime}} j} .
$$

To derive the explicit expressions for the expected value and risk of $\widetilde{\beta}_{N_{m}^{\prime}}$, let $\mathcal{F}_{m}=\sigma\left(X_{1}, X_{2}, \ldots, X_{m}\right)$ be the $\sigma$-field generated by the random variables at the first stage. Then we have

I. $\bar{S}_{m}=\frac{S_{m}}{m \alpha}$ is measurable in $\mathcal{F}_{m}$.

II. $N_{m}^{\prime}$ is measurable in $\mathcal{F}_{m}$.

III. $\bar{S}_{N_{m}^{\prime}-m}$ is independent of $\mathcal{F}_{m}$.

The following theorem gives the expression for exact risk of $\widetilde{\beta}_{N_{m}^{\prime}}$. 
Theorem 2.4. The risk of $\widetilde{\beta}_{N_{m}^{\prime}}$ is

$$
\begin{aligned}
R\left(\widetilde{\beta}_{N_{m}^{\prime}}, \beta\right)= & A \beta^{2}\left[1+\frac{(m \alpha+1)}{m \alpha} G\left(\lambda_{m}^{\prime} ; m \alpha+2,1\right)\right. \\
& +\sum_{n=m+1}^{\infty} \frac{m \alpha(m \alpha+1)}{(n \alpha)^{2}}\left\{\bar{G}\left(\lambda_{n-1}^{\prime} ; m \alpha+2,1\right)-\bar{G}\left(\lambda_{n}^{\prime} ; m \alpha+2,1\right)\right\} \\
& +\sum_{n=m+1}^{\infty} \frac{(n-m)}{n^{2} \alpha}\left\{\bar{G}\left(\lambda_{n-1}^{\prime} ; m \alpha, 1\right)-\bar{G}\left(\lambda_{n}^{\prime} ; m \alpha, 1\right)\right\} \\
& +\sum_{n=m+1}^{\infty}\left(\frac{(n-m)}{n}\right)^{2}\left\{\bar{G}\left(\lambda_{n-1}^{\prime} ; m \alpha, 1\right)-\bar{G}\left(\lambda_{n}^{\prime} ; m \alpha, 1\right)\right\} \\
& +\sum_{n=m+1}^{\infty} \frac{2 m(n-m)}{n^{2}}\left\{\bar{G}\left(\lambda_{n-1}^{\prime} ; m \alpha+1,1\right)-\bar{G}\left(\lambda_{n}^{\prime} ; m \alpha+1,1\right)\right\}-2 G\left(\lambda_{m}^{\prime} ; m \alpha+1,1\right) \\
& -\sum_{n=m+1}^{\infty} \frac{2 m}{n}\left\{\bar{G}\left(\lambda_{n-1}^{\prime} ; m \alpha+1,1\right)-\bar{G}\left(\lambda_{n}^{\prime} ; m \alpha+1,1\right)\right\} \\
& \left.-\sum_{n=m+1}^{\infty} \frac{2(n-m)}{n}\left\{\bar{G}\left(\lambda_{n-1}^{\prime} ; m \alpha, 1\right)-\bar{G}\left(\lambda_{n}^{\prime} ; m \alpha, 1\right)\right\}\right] .
\end{aligned}
$$

One may refer to the Appendix for the proofs of the theorems in this section.

\subsection{Comparison of the Risk of Estimators of $\beta$}

In this section, we compare the risk of the MMSE of parameter $\beta$ of $\operatorname{Gamma}(\alpha, \beta)$ distribution with the risk of the MLE/UMVUE of $\beta$. We consider different pilot sample sizes at the first stage, i.e. $m$. Fixing the weight of the risk function $A$ and $n^{*}$, we calculate the upper bound of the risk function $\omega$, which is further used to obtain $n^{* *}$. Using these values we compute the expressions for the exact risk of $\widehat{\beta}_{N_{m}}$ and $\widetilde{\beta}_{N_{m}^{\prime}}$. We also compare the estimates of the exact risk of MMSE and MLE/UMVUE of $\beta$ obtained through simulations. For each $m$, we estimate the exact risk of MMSE of $\beta$ denoted by $\overline{R\left(\widehat{\beta}_{N_{m}}, \beta\right)}$ and the exact risk of MLE/UMVUE of $\beta$ denoted by $\overline{R\left(\widetilde{\beta}_{N_{m}^{\prime}}, \beta\right)}$ over 10,000 replications. We also obtain the standard error of the exact true risk of MMSE and MLE/UMVUE of $\beta$. The following tables summarise our numerical findings in case of Gamma distribution for different values of the parameters. For different pilot sample sizes $m$, we compute observed sample mean of the MMSE of $\beta$ denoted by $\overline{\widehat{\beta}_{m}}$ and its 
standard error. Similarly we also compute the observed sample mean of the MLE/UMVUE of $\beta$ denoted by $\widetilde{\widetilde{\beta}_{m}}$ along with its standard error. In the following tables, for each $m$, the values just below the values of $\overline{\widehat{\beta}_{m}}$, $\overline{R\left(\widehat{\beta}_{N_{m}}, \beta\right)}, \widetilde{\beta}_{m}$ and $\overline{R\left(\widetilde{\beta}_{N_{m}^{\prime}}, \beta\right)}$ are their respective standard errors.

Table 2.1: Comparison of exact risk of $\widehat{\beta}_{N_{m}}$ and $\widetilde{\beta}_{N_{m}^{\prime}}$ under the two-stage procedures (2.1) and (2.2) respectively when $\alpha=4, \beta=3, A=5, n^{*}=400$, $n^{* *}=400.25$ and $\omega=0.02810743$.

\begin{tabular}{|c|c|c|c|c|c|c|c|c|c|c|}
\hline$m$ & $\widehat{\widehat{\beta}_{N_{m}}}$ & $\overline{N_{m}}$ & $B$ & $R\left(\widehat{\beta}_{N_{m}}, \beta\right)$ & $\overline{R\left(\widehat{\beta}_{N_{m}}, \beta\right)}$ & $\widetilde{\beta}_{N_{m}^{\prime}}$ & $\overline{N_{m}^{\prime}}$ & $B^{\prime}$ & $R\left(\widetilde{\beta}_{N_{m}^{\prime}}, \beta\right)$ & $\overline{R\left(\widetilde{\beta}_{N_{m}^{\prime}}, \beta\right)}$ \\
\hline \multirow[t]{2}{*}{10} & 2.99769 & 924.74880 & 11.76813 & 0.01363 & 0.01384 & 2.99823 & 913.85810 & 11.06613 & 0.01376 & 0.01385 \\
\hline & 0.00053 & 2.93138 & & & 0.00022 & 0.00053 & 2.89613 & & & 0.00021 \\
\hline \multirow[t]{2}{*}{15} & 2.99715 & 880.18460 & 11.14560 & 0.01379 & 0.01353 & 2.99761 & 873.28100 & 10.69550 & 0.01387 & 0.01375 \\
\hline & 0.00052 & 2.26776 & & & 0.00020 & 0.00052 & 2.24938 & & & 0.00020 \\
\hline
\end{tabular}

Table 2.2: Comparison of exact risk of $\widehat{\beta}_{N_{m}}$ and $\widetilde{\beta}_{N_{m}^{\prime}}$ under the two-stage procedures (2.1) and 2.2 respectively when $\alpha=4, \beta=3, A=5, n^{*}=500$, $n^{* *}=500.25$ and $\omega=0.02248876$.

\begin{tabular}{|c|c|c|c|c|c|c|c|c|c|c|}
\hline$m$ & $\overline{\widehat{\beta}_{N_{m}}}$ & $\overline{N_{m}}$ & $B$ & $R\left(\widehat{\beta}_{N_{m}}, \beta\right)$ & $\overline{R\left(\widehat{\beta}_{N_{m}}, \beta\right)}$ & $\widetilde{\beta}_{N_{m}^{\prime}}$ & $\overline{N_{m}^{\prime}}$ & $B^{\prime}$ & $R\left(\widetilde{\beta}_{N_{m}^{\prime}}, \beta\right)$ & $R\left(\widetilde{\beta}_{N_{m}^{\prime}}, \beta\right)$ \\
\hline \multirow[t]{2}{*}{10} & 2.99820 & 1155.80100 & 11.76813 & 0.01089 & 0.01070 & 2.99794 & 1142.12000 & 11.06613 & 0.01100 & 0.01075 \\
\hline & 0.00046 & 3.68300 & & & 0.00017 & 0.00046 & 3.63864 & & & 0.00016 \\
\hline \multirow[t]{2}{*}{15} & 2.99809 & 1097.47500 & 11.14560 & 0.01102 & 0.01125 & 2.99861 & 1088.79700 & 10.69550 & 0.01109 & 0.01131 \\
\hline & 0.00047 & 2.83406 & & & 0.00017 & 0.00048 & 2.81098 & & & 0.00017 \\
\hline
\end{tabular}

Table 2.3: Comparison of exact risk of $\widehat{\beta}_{N_{m}}$ and $\widetilde{\beta}_{N_{m}^{\prime}}$ under the two-stage procedures (2.1) and (2.2) respectively when $\alpha=4, \beta=3, A=5, n^{*}=600$, $n^{* *}=600.25$ and $\omega=0.01874219$.

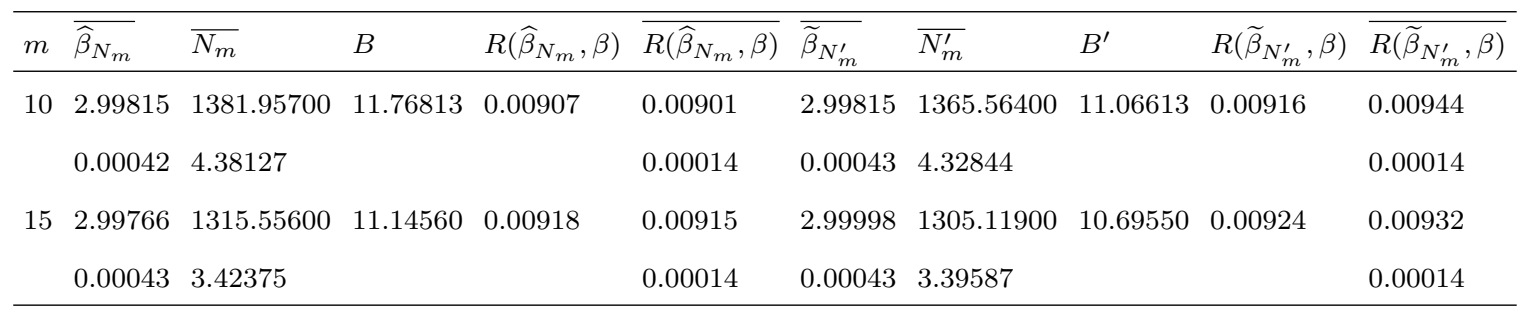

From the above tables we conclude that irrespective of the values of the parameters $\alpha, \beta, A, n^{*}, n^{* *}$ and $\omega$, the exact risk of the MMSE of $\beta$ is always lower than the exact risk of the MLE/ UMVUE of $\beta$. Thus, we are able to establish that even though biased, the MMSE of $\beta$ has a smaller risk and 
hence is a better choice over the MLE/ UMVUE of $\beta$. It is interesting to note that the risk of two-stage procedure is too small as compared to the target value $\omega$. Thus the two-stage procedure itself reduces the risk drastically.

\section{Estimation of Hazard Rate of $F_{2}$}

\subsection{Two-Stage Procedure based on Minimum Mean Square Estimator}

Let $X_{1}, X_{2}, \ldots, X_{m}$ be a pilot sample from the family of distributions $\boldsymbol{F}_{\mathbf{2}}$. Using Stein's two-stage sampling scheme [see Stein $(1945,1949)]$, we propose the following stopping rule:

$$
N_{m}=N\left(m, K, \omega^{\prime}\right)=\max \left\{m,\left\lfloor\frac{K\left(\theta g^{\theta-1}(t) g^{\prime}(t)\right)^{2}}{\omega^{\prime} \bar{S}_{m}^{2}}+1\right\rfloor+1\right\},
$$

where $\lfloor z\rfloor$ denotes the greatest integer less than $z . K$ is a positive coefficient and is determined such that the risk $R\left(\widehat{\gamma}_{N_{m}}, \gamma\right)$ is bounded by a pre-assigned number $\omega^{\prime} . \bar{S}_{m}=\frac{S_{m}}{m-2}=\frac{\sum_{i=1}^{m} g^{\theta}\left(x_{i}\right)}{m-2}$. We shall later prove that $K$ is a function of all the known quantities $A^{\prime}$ and $m$. Now, if $N_{m}=m$, then the pilot sample is large enough and we don't require to draw more observations at the second stage. But if $N_{m}>m$, then the pilot sample is not large enough and hence we must draw $N_{m}-m$ more observations at the second stage, say $X_{m+1}, X_{m+2}, \ldots, X_{N_{m}}$. Finally based on all the observations from both the stages, $X_{1}, X_{2}, \ldots, X_{N_{m}}$, we estimate the parameter $\gamma$ by its MMSE, i.e.

$$
\widehat{\gamma}_{N_{m}}=\frac{\theta g^{\theta-1}(t) g^{\prime}(t)}{\left(\frac{\sum_{i=1}^{N_{m}} g^{\theta}\left(x_{i}\right)}{N_{m}-2}\right)} .
$$

Thus the risk associated with this estimator is

$$
R\left(\widehat{\gamma}_{N_{m}}, \gamma\right)=A^{\prime} E\left(\left(\widehat{\gamma}_{N_{m}}-\gamma\right)^{2}\right)
$$

Theorem 3.1. Consider the two-stage procedure in (3.1) and the loss function in 1.15) for sample size $N_{m}$. If we estimate $\gamma$ by $\widehat{\gamma}_{N_{m}}$, then for all fixed $\nu, \theta, t, m$ and $A^{\prime}$, we conclude that $R\left(\widehat{\gamma}_{N_{m}}, \gamma\right) \leq \omega^{\prime}$ and

$$
K=\frac{A^{\prime}\left(\theta g^{\theta-1}(t) g^{\prime}(t)\right)^{2}(2 m+7)}{m+1} .
$$


The $r v N_{m}$ in (3.1) is a discrete $r v$ and can take values $\{m, m+1, \ldots\}$. We define

$$
\lambda_{j}=\left(\frac{m+1}{\nu}\right) \sqrt{\frac{K\left(\theta g^{\theta-1}(t) g^{\prime}(t)\right)^{2}}{\omega^{\prime}(j+1)}}
$$

and $G(z ; a, b)$ as the cumulative distribution function $(c d f)$ of gamma distribution at the point $z$ with shape parameter $a$ and scale parameter $b$. Also let $\bar{G}(z ; a, b)=1-G(z ; a, b)$.

To derive the explicit expressions for the expected value of $\widehat{\nu}_{N_{m}}$, let $\mathcal{F}_{m}=\sigma\left(X_{1}, X_{2}, \ldots, X_{m}\right)$ be the $\sigma$-field generated by the random variables at the first stage. Then we have

I. $\bar{S}_{m}=\frac{S_{m}}{m-2}$ is measurable in $\mathcal{F}_{m}$.

II. $N_{m}$ is measurable in $\mathcal{F}_{m}$.

III. $\bar{S}_{N_{m}-m}$ is independent of $\mathcal{F}_{m}$.

The following theorem gives the expression for exact risk of $\widehat{\gamma}_{N_{m}}$.

Theorem 3.2. The risk of $\widehat{\gamma}_{N_{m}}$ is

$$
\begin{aligned}
R\left(\widehat{\gamma}_{N_{m}}, \gamma\right)= & \frac{A^{\prime}\left(\theta g^{\theta-1}(t) g^{\prime}(t)\right)^{2}}{\nu^{2}}\left[1+\frac{m}{(m+1)} \bar{G}\left(\lambda_{m} ; m+2,1\right)\right. \\
& +\sum_{n=m+1}^{\infty} \frac{m(m+1)}{(n+1)^{2}}\left\{G\left(\lambda_{n-1} ; m+2,1\right)-G\left(\lambda_{n} ; m+2,1\right)\right\} \\
& +\sum_{n=m+1}^{\infty} \frac{(n-m)}{(n+1)^{2}}\left\{G\left(\lambda_{n-1} ; m, 1\right)-G\left(\lambda_{n} ; m, 1\right)\right\} \\
& +\sum_{n=m+1}^{\infty}\left(\frac{n-m}{n+1}\right)^{2}\left\{G\left(\lambda_{n-1} ; m, 1\right)-G\left(\lambda_{n} ; m, 1\right)\right\} \\
& +\sum_{n=m+1}^{\infty} \frac{2 m(n-m)}{(n+1)^{2}}\left\{G\left(\lambda_{n-1} ; m+1,1\right)-G\left(\lambda_{n} ; m+1,1\right)\right\} \\
& -2 \frac{m}{m+1} \bar{G}\left(\lambda_{m} ; m+1,1\right) \\
& -\sum_{n=m+1}^{\infty} \frac{2 m}{n+1}\left\{G\left(\lambda_{n-1} ; m+1,1\right)-G\left(\lambda_{n} ; m+1,1\right)\right\} \\
& \left.-\sum_{n=m+1}^{\infty} \frac{2(n-m)}{n+1}\left\{G\left(\lambda_{n-1} ; m, 1\right)-G\left(\lambda_{n} ; m, 1\right)\right\}\right]
\end{aligned}
$$




\subsection{Two-Stage Procedure based on Uniformly Mini- mum Variance Unbiased Estimator}

Let $X_{1}, X_{2}, \ldots, X_{m}$ be a pilot sample from the family of distributions $\boldsymbol{F}_{\mathbf{2}}$. Using Stein's two-stage sampling scheme [see Stein $(1945,1949)]$, we propose the following stopping rule:

$$
N_{m}^{\prime}=N\left(m, K^{\prime}, \omega^{\prime}\right)=\max \left\{m,\left\lfloor\frac{K^{\prime}\left(\theta g^{\theta-1}(t) g^{\prime}(t)\right)^{2}}{\omega^{\prime} \bar{S}_{m}^{2}}+2\right\rfloor+1\right\},
$$

where $K^{\prime}$ is a positive coefficient and is determined such that the risk of UMVUE of the hazard rate $R\left(\widetilde{\gamma}_{N_{m}^{\prime}}, \gamma\right)$ is bounded by a pre-assigned number $\omega^{\prime} . \bar{S}_{m}=\frac{S_{m}}{m-1}=\frac{\sum_{i=1}^{m} g^{\theta}\left(x_{i}\right)}{m-1}$. Based on all the observations from both the stages, $X_{1}, X_{2}, \ldots, X_{N_{m}^{\prime}}$, we estimate the parameter $\gamma$ by its UMVUE, i.e.

$$
\widetilde{\gamma}_{N_{m}^{\prime}}=\frac{\theta g^{\theta-1}(t) g^{\prime}(t)}{\left(\frac{\sum_{i=1}^{N_{m}^{\prime}} g^{\theta}\left(x_{i}\right)}{N_{m}^{\prime}-1}\right)}
$$

Thus the risk associated with this estimator is

$$
R\left(\widetilde{\gamma}_{N_{m}^{\prime}}, \gamma\right)=A^{\prime} E\left(\left(\widetilde{\gamma}_{N_{m}^{\prime}}-\gamma\right)^{2}\right)
$$

Theorem 3.3. Consider the two-stage procedure in (3.2) and the loss function in (1.20) for sample size $N_{m}^{\prime}$. If we estimate $\gamma$ by $\widetilde{\gamma}_{N_{m}^{\prime}}$, then for all fixed $\nu, \theta, t, m$ and $A^{\prime}$, we conclude that $R\left(\widetilde{\gamma}_{N_{m}^{\prime}}, \gamma\right) \leq \omega^{\prime}$ and

$$
K^{\prime}=\frac{2 A^{\prime}\left(\theta g^{\theta-1}(t) g^{\prime}(t)\right)^{2}(m+1)(m+3)}{m^{2}} .
$$

The $r v N_{m}^{\prime}$ in $(3.2)$ is a discrete $r v$ and can take values $\{m, m+1, \ldots\}$. We define

$$
\lambda_{j}^{\prime}=\left(\frac{m}{\nu}\right) \sqrt{\frac{K^{\prime}\left(\theta g^{\theta-1}(t) g^{\prime}(t)\right)^{2}}{\omega^{\prime} j}} .
$$

To derive the explicit expressions for the expected value of $\widetilde{\nu}_{N_{m}^{\prime}}$, let $\mathcal{F}_{m}=\sigma\left(X_{1}, X_{2}, \ldots, X_{m}\right)$ be the $\sigma$-field generated by the random variables at the first stage. Then we have

I. $\bar{S}_{m}=\frac{S_{m}}{m}$ is measurable in $\mathcal{F}_{m}$.

II. $N_{m}^{\prime}$ is measurable in $\mathcal{F}_{m}$.

III. $\bar{S}_{N_{m}^{\prime}-m}$ is independent of $\mathcal{F}_{m}$. 
The following theorem gives the expression for exact risk of $\widetilde{\gamma}_{N_{m}^{\prime}}$.

Theorem 3.4. The risk of $\widetilde{\gamma}_{N_{m}^{\prime}}$ is

$$
\begin{aligned}
R\left(\widetilde{\gamma}_{N_{m}^{\prime}}, \gamma\right)= & \frac{A^{\prime}\left(\theta g^{\theta-1}(t) g^{\prime}(t)\right)^{2}}{\nu^{2}}\left[1+\frac{(m+1)}{m} \bar{G}\left(\lambda_{m}^{\prime} ; m+2,1\right)\right. \\
& +\sum_{n=m+1}^{\infty} \frac{m(m+1)}{n^{2}}\left\{G\left(\lambda_{n-1}^{\prime} ; m+2,1\right)-G\left(\lambda_{n}^{\prime} ; m+2,1\right)\right\} \\
& +\sum_{n=m+1}^{\infty} \frac{(n-m)}{n^{2}}\left\{G\left(\lambda_{n-1}^{\prime} ; m, 1\right)-G\left(\lambda_{n}^{\prime} ; m, 1\right)\right\} \\
& +\sum_{n=m+1}^{\infty}\left(\frac{n-m}{n}\right)^{2}\left\{G\left(\lambda_{n-1}^{\prime} ; m, 1\right)-G\left(\lambda_{n}^{\prime} ; m, 1\right)\right\} \\
& +\sum_{n=m+1}^{\infty} \frac{2 m(n-m)}{n^{2}}\left\{G\left(\lambda_{n-1}^{\prime} ; m+1,1\right)-G\left(\lambda_{n}^{\prime} ; m+1,1\right)\right\}-2 \bar{G}\left(\lambda_{m}^{\prime} ; m+1,1\right) \\
& -\sum_{n=m+1}^{\infty} \frac{2 m}{n}\left\{G\left(\lambda_{n-1}^{\prime} ; m+1,1\right)-G\left(\lambda_{n}^{\prime} ; m+1,1\right)\right\} \\
& \left.-\sum_{n=m+1}^{\infty} \frac{2(n-m)}{n}\left\{G\left(\lambda_{n-1}^{\prime} ; m, 1\right)-G\left(\lambda_{n}^{\prime} ; m, 1\right)\right\}\right]
\end{aligned}
$$

One may refer to the Appendix for the proofs of the theorems in this section.

\subsection{Comparison of the Risk of Estimators of Hazard Rate}

In this section, we compare the risk of the estimator of the hazard rate function $\gamma=\frac{\theta g^{\theta-1}(t) g^{\prime}(t)}{\nu}$ of $W$ eibull $(\theta, \nu)$ distribution when it is estimated by its MMSE with the risk of the estimator of $\gamma$ when it is estimated by its UMVUE. We consider different sample sizes at the first stage, i.e. $m$. Fixing the weight of the risk function $A^{\prime}$ and $n^{\prime *}$ we calculate the upper bound of the risk function $\omega^{\prime}$, which is further used to obtain $n^{\prime * *}$. Using these values we compute the expressions for the exact risk of $\widehat{\gamma}_{N_{m}}$ and $\widetilde{\gamma}_{N_{m}^{\prime}}$. We also compare the estimates of the exact risk of MMSE and UMVUE of $\gamma$ obtained through simulations. For each $m$, we estimate the exact risk of MMSE of $\gamma$ denoted by $\overline{R\left(\widehat{\gamma}_{N_{m}}, \gamma\right)}$ and the exact true risk of UMVUE of $\gamma$ denoted by $\overline{R\left(\widetilde{\gamma}_{N_{m}^{\prime}}, \gamma\right)}$ over 10,000 replications. We also obtain the standard error of the exact risk of MMSE and UMVUE of $\gamma$. The following tables summarise our numerical 
findings in case of Weibull distribution for different values of the parameters. For different pilot sample sizes $m$, we compute observed sample mean of the MMSE of $\gamma$ denoted by $\overline{\widehat{\gamma}_{m}}$ and its standard error. Similarly we also compute the observed sample mean of the UMVUE of $\gamma$ denoted by $\widetilde{\gamma}_{m}$ along with its standard error. In the following tables, for each $m$, the values just below the values of $\overline{\widehat{\gamma}_{m}}, \overline{R\left(\widehat{\gamma}_{N_{m}}, \gamma\right)}, \overline{\widetilde{\beta}_{m}}$ and $\overline{R\left(\widetilde{\gamma}_{N_{m}^{\prime}}, \gamma\right)}$ are their respective standard errors.

Table 3.1: Comparison of exact risk of $\widehat{\gamma}_{N_{m}}$ and $\widetilde{\gamma}_{N_{m}^{\prime}}$ under the two-stage procedures (3.1) and (3.2) respectively when $\theta=1, \nu=2, A^{\prime}=10, n^{*}=10$, $n^{* *}=11, \omega^{\prime}=0.2777778$ and when time is $t=20, \gamma=0.5$.

\begin{tabular}{|ccccccccccc|}
\hline$m$ & $\overline{\widehat{\gamma}_{N_{m}}}$ & $\overline{N_{m}}$ & $K$ & $R\left(\widehat{\gamma}_{N_{m}}, \gamma\right)$ & $\overline{R\left(\widehat{\gamma}_{N_{m}}, \gamma\right)}$ & $\overline{\widetilde{\gamma}_{N_{m}^{\prime}}}$ & $\overline{N_{m}^{\prime}}$ & $K^{\prime}$ & $R\left(\widetilde{\gamma}_{N_{m}^{\prime}}, \gamma\right)$ & $\overline{R\left(\widetilde{\gamma}_{N_{m}^{\prime}}, \gamma\right)}$ \\
\hline 10 & 0.42572 & 20.06100 & 24.54545 & 0.11540 & 0.10547 & 0.46835 & 31.69830 & 28.60000 & 0.16162 & 0.11000 \\
& 0.00117 & 0.16350 & & & 0.00308 & 0.00100 & 0.24898 & & & 0.00158 \\
15 & 0.44439 & 20.77710 & 23.12500 & 0.09468 & 0.09716 & 0.47129 & 27.56810 & 25.60000 & 0.12695 & 0.10309 \\
& 0.00101 & 0.10359 & & & 0.00245 & 0.00097 & 0.14795 & & & 0.00130 \\
\hline
\end{tabular}

Table 3.2: Comparison of exact risk of $\widehat{\gamma}_{N_{m}}$ and $\widetilde{\gamma}_{N_{m}^{\prime}}$ under the two-stage procedures (3.1) and (3.2) respectively when $\theta=1, \nu=2, A^{\prime}=10, n^{*}=13$, $n^{* *}=14, \omega^{\prime}=0.2083333$ and when time is $t=20, \gamma=0.5$.

\begin{tabular}{|ccccccccccc|}
\hline$m$ & $\overline{\gamma_{N_{m}}}$ & $\overline{N_{m}}$ & $K$ & $R\left(\widehat{\gamma}_{N_{m}}, \gamma\right)$ & $\overline{R\left(\widehat{\gamma}_{N_{m}}, \gamma\right)}$ & $\widetilde{\gamma}_{N_{m}^{\prime}}$ & $\overline{N_{m}^{\prime}}$ & $K^{\prime}$ & $R\left(\widetilde{\gamma}_{N_{m}^{\prime}}, \gamma\right)$ & $\overline{R\left(\widetilde{\gamma}_{N_{m}^{\prime}}, \gamma\right)}$ \\
\hline 10 & 0.43574 & 26.24300 & 24.54545 & 0.09676 & 0.08192 & 0.47299 & 41.44950 & 28.60000 & 0.12804 & 0.09097 \\
& 0.00112 & 0.21339 & & & 0.00218 & 0.00091 & 0.31862 & & & 0.00142 \\
15 & 0.44775 & 26.21010 & 23.12500 & 0.08942 & 0.07905 & 0.47545 & 35.77010 & 25.60000 & 0.11652 & 0.08758 \\
& 0.00099 & 0.14459 & & & 0.00196 & 0.00090 & 0.19474 & & & 0.00119 \\
\hline
\end{tabular}

From the above tables we conclude that irrespective of the values of the parameters, the exact risk of the MMSE of the hazard rate function $\gamma$ is always lower than the exact risk of the UMVUE of $\gamma$. Thus, we are able to establish that even though biased, the MMSE of $\gamma$ has a smaller risk and hence is a better choice over the UMVUE of $\gamma$. Also, we may note that the risk of two-stage procedure is too small as compared to the target value $\omega^{\prime}$. Thus the two-stage procedure itself reduces the risk drastically. 
Table 3.3: Comparison of exact risk of $\widehat{\gamma}_{N_{m}}$ and $\widetilde{\gamma}_{N_{m}^{\prime}}$ under the two-stage procedures (3.1) and (3.2) respectively when $\theta=1, \nu=2, A^{\prime}=10, n^{*}=15$, $n^{* *}=16, \omega^{\prime}=0.1785714$ and when time is $t=20, \gamma=0.5$.

\begin{tabular}{|ccccccccccc|}
\hline$m$ & $\overline{\widehat{\gamma}_{N_{m}}}$ & $\overline{N_{m}}$ & $K$ & $R\left(\widehat{\gamma}_{N_{m}}, \gamma\right)$ & $\overline{R\left(\widehat{\gamma}_{N_{m}}, \gamma\right)}$ & $\overline{\widetilde{\gamma}_{N_{m}^{\prime}}}$ & $\overline{N_{m}^{\prime}}$ & $K^{\prime}$ & $R\left(\widetilde{\gamma}_{N_{m}^{\prime}}, \gamma\right)$ & $\overline{R\left(\widetilde{\gamma}_{N_{m}^{\prime}}, \gamma\right)}$ \\
\hline 10 & 0.440397 & 30.213800 & 24.545450 & 0.084764 & 0.073477 & 0.477359 & 47.528600 & 28.600000 & 0.109034 & 0.079129 \\
& 0.001082 & 0.266649 & & & 0.001974 & 0.000860 & 0.395400 & & & 0.001244 \\
15 & 0.450610 & 30.043100 & 23.125000 & 0.083130 & 0.070099 & 0.477028 & 41.097200 & 25.600000 & 0.105750 & 0.079533 \\
& 0.000974 & 0.177012 & & & 0.001711 & 0.000862 & 0.233141 & & & 0.001145 \\
\hline
\end{tabular}

\section{Appendix}

Proof of Theorem 2.1. Let $\mathcal{F}_{m}=\sigma\left(X_{1}, X_{2}, \ldots, X_{m}\right)$ be the $\sigma$-field generated by the sample $X_{1}, X_{2}, \ldots, X_{m}$ at the first stage, then $\mathcal{F}_{m}=\frac{S_{m}}{m \alpha}$ and $N_{m}$ measurable with respect to $\mathcal{F}_{m}$ and $\tilde{S}_{N_{m}-m}=\frac{\sum_{i=m+1}^{N_{m}} g\left(x_{i}, \boldsymbol{\theta}\right)}{\left(N_{m}-m\right) \alpha}$ is independent of $\mathcal{F}_{m}$. Consider,

$$
\begin{aligned}
R\left(\widehat{\beta}_{N_{m}}, \beta\right)= & E\left(A\left(\widehat{\beta}_{N_{m}}-\beta\right)^{2}\right) \\
= & A E\left[E\left(\left(\frac{m \alpha \tilde{S}_{m}+\left(N_{m}-m\right) \alpha \tilde{S}_{N_{m}-m}}{N_{m} \alpha+1}-\beta\right)^{2} \mid \mathcal{F}_{m}\right)\right] \\
= & \frac{A}{\alpha^{2}}\left[E\left(\frac{m^{2} \alpha^{2}\left(\tilde{S}_{m}-\beta\right)^{2}}{\left(N_{m}+\frac{1}{\alpha}\right)^{2}}\right)\right. \\
& \left.+E\left(\frac{\left(N_{m}-m\right)^{2} \alpha^{2}}{\left(N_{m}+\frac{1}{\alpha}\right)^{2}} E\left(\left(\tilde{S}_{N_{m}-m}-\beta\right)^{2} \mid \mathcal{F}_{m}\right)\right)+E\left(\frac{\beta^{2}}{\left(N_{m}+\frac{1}{\alpha}\right)^{2}}\right)\right] \\
= & \frac{A}{\alpha^{2}}\left[J_{1}+J_{2}+J_{3}\right] .
\end{aligned}
$$

Now we consider the following inequalities:

$$
\frac{m^{2}}{\left(N_{m}+\frac{1}{\alpha}\right)^{2}} \leq \frac{m}{N_{m}+\frac{1}{\alpha}}, \quad \frac{1}{N_{m}+\frac{1}{\alpha}} \leq \frac{\alpha \omega}{B \bar{S}_{m}^{2}} .
$$

We can now derive the bounds on $J_{1}, J_{2}$ and $J_{3}$ in (A.1) by using the inequalities in (A.2.

$$
J_{1}=E\left(\frac{m^{2} \alpha^{2}\left(\tilde{S}_{m}-\beta\right)^{2}}{\left(N_{m}+\frac{1}{\alpha}\right)^{2}}\right) \leq E\left(\frac{m \alpha^{2}\left(\tilde{S}_{m}-\beta\right)^{2}}{\left(N_{m}+\frac{1}{\alpha}\right)}\right)
$$




$$
\begin{aligned}
& \leq E\left(\frac{m \alpha^{3} \omega\left(\tilde{S}_{m}-\beta\right)^{2}}{B \bar{S}_{m}^{2}}\right) \\
& =E\left(\frac{m \alpha^{3} \omega}{B}\left(\left(\frac{m \alpha+1}{m \alpha}\right)^{2}+\frac{\beta^{2}}{\bar{S}_{m}^{2}}-\frac{2 \beta}{\bar{S}_{m}}\left(\frac{m \alpha+1}{m \alpha}\right)\right)\right) .
\end{aligned}
$$

Since $\left(\frac{m \alpha+1}{\beta}\right) \bar{S}_{m} \sim \operatorname{Gamma}(m \alpha, 1)$, thus

$$
J_{1} \leq \frac{m \alpha^{3} \omega}{B}\left[\left(\frac{m \alpha+1}{m \alpha}\right)^{2}+\frac{(m \alpha+1)^{2}}{(m \alpha-1)(m \alpha-2)}-2 \frac{(m \alpha+1)^{2}}{m \alpha(m \alpha-1)}\right]
$$

Now, since $\tilde{S}_{N_{m}-m} \sim \operatorname{Gamma}\left(\left(N_{m}-m\right) \alpha, \frac{\beta}{\left(N_{m}-m\right) \alpha}\right)$,

$$
\begin{aligned}
J_{2} & =\alpha \beta^{2} E\left(\frac{N_{m}-m}{\left(N_{m}+\frac{1}{\alpha}\right)^{2}}\right) \leq \alpha \beta^{2} E\left(\frac{N_{m}-m}{m\left(N_{m}+\frac{1}{\alpha}\right)}\right) \\
& \leq \alpha \beta^{2} E\left(\left(\frac{N_{m}}{m}-1\right) \frac{1}{\left(N_{m}+\frac{1}{\alpha}\right)} I\left(N_{m}>m\right)\right) \\
& \leq \alpha \beta^{2} E\left(\frac{1}{\left(N_{m}+\frac{1}{\alpha}\right)}\right) \\
& \leq \frac{\alpha^{2} \omega}{B} \frac{(m \alpha+1)^{2}}{(m \alpha-1)(m \alpha-2)} .
\end{aligned}
$$

Similarly we can obtain

$$
J_{3} \leq \frac{\alpha \omega}{B m} \frac{(m \alpha+1)^{2}}{(m \alpha-1)(m \alpha-2)} .
$$

Substituting A.3 , A.4 and A.5 in A.1 and using $R\left(\widehat{\beta}_{N_{m}}, \beta\right) \leq \omega$, it is sufficient that

$$
B=\frac{A(m \alpha+1)^{2}(2 m \alpha+3)}{m \alpha(m \alpha-1)(m \alpha-2)} .
$$

Proof of Theorem 2.2.

$$
\begin{aligned}
R\left(\widehat{\beta}_{N_{m}}, \beta\right) & =E\left(A\left(\widehat{\beta}_{N_{m}}-\beta\right)^{2}\right) \\
& =A\left[E\left(\widehat{\beta}_{N_{m}}^{2}\right)+\beta^{2}-2 \beta E\left(\widehat{\beta}_{N_{m}}\right)\right],
\end{aligned}
$$


where we derive expressions for $E\left(\widehat{\beta}_{N_{m}}\right)$ and $E\left(\widehat{\beta}_{N_{m}}^{2}\right)$ using the same techniques as adopted by Mahmoudi and Roughani (2015) and Roughani and Mahmoudi (2015). On similar lines we can also derive the results of Theorems 2.3 and 2.4 .

Proof of Theorem 3.1. Let $\mathcal{F}_{m}=\sigma\left(X_{1}, X_{2}, \ldots, X_{m}\right)$ be the $\sigma$-field generated by the sample $X_{1}, X_{2}, \ldots, X_{m}$ at the first stage, then $\tilde{S}_{m}=\frac{S_{m}}{m}$ and $N_{m}$ measurable with respect to $\mathcal{F}_{m}$ and $\tilde{S}_{N_{m}-m}=\frac{\sum_{i=m+1}^{N_{m}} g^{\theta}\left(x_{i}\right)}{\left(N_{m}-m\right)}$ is independent of $\mathcal{F}_{m}$. Consider,

$$
R\left(\widehat{\gamma}_{N_{m}}, \gamma\right)=E\left(A^{\prime}\left(\widehat{\gamma}_{N_{m}}-\gamma\right)^{2}\right),
$$

which on applying Taylor's series approximation gives

$$
\begin{aligned}
R\left(\widehat{\gamma}_{N_{m}}, \gamma\right) & =\frac{A^{\prime}\left(\theta g^{\theta-1}(t) g^{\prime}(t)\right)^{2}}{\nu^{4}} E\left(\left(\widehat{\nu}_{N_{m}}-\nu\right)^{2}\right) \\
& =\frac{A^{\prime}\left(\theta g^{\theta-1}(t) g^{\prime}(t)\right)^{2}}{\nu^{4}} E\left[E\left(\left(\frac{m \tilde{S}_{m}+\left(N_{m}-m\right) \tilde{S}_{N_{m}-m}}{N_{m}+1}-\nu\right)^{2} \mid \mathcal{F}_{m}\right)\right] \\
& =\frac{A^{\prime}\left(\theta g^{\theta-1}(t) g^{\prime}(t)\right)^{2}}{\nu^{4}}\left[E\left(\frac{m^{2}\left(\tilde{S}_{m}-\nu\right)^{2}}{\left(N_{m}+1\right)^{2}}\right)\right. \\
& \left.+E\left(\frac{\left(N_{m}-m\right)^{2}}{\left(N_{m}+1\right)^{2}} E\left(\left(\tilde{S}_{N_{m}-m}-\nu\right)^{2} \mid \mathcal{F}_{m}\right)\right)+E\left(\frac{\nu^{2}}{\left(N_{m}+1\right)^{2}}\right)\right] \\
\nu^{4} & \frac{A^{\prime}\left(\theta g^{\theta-1}(t) g^{\prime}(t)\right)^{2}}{(\mathrm{~A} .6)}
\end{aligned}
$$

Now we consider the following inequalities:

$$
\frac{m^{2}}{\left(N_{m}+1\right)^{2}} \leq \frac{m}{N_{m}+1}, \frac{1}{N_{m}+1} \leq \frac{\omega^{\prime} \bar{S}_{m}^{2}}{K} .
$$

We can now derive the bounds on $J_{1}, J_{2}$ and $J_{3}$ in A.6 by using the inequalities in (A.7).

$$
\begin{aligned}
J_{1} & =E\left(\frac{m^{2}\left(\tilde{S}_{m}-\nu\right)^{2}}{\left(N_{m}+1\right)^{2}}\right) \leq E\left(\frac{m\left(\tilde{S}_{m}-\nu\right)^{2}}{\left(N_{m}+1\right)}\right) \\
& \leq E\left(\frac{m \omega^{\prime} \bar{S}_{m}^{2}\left(\tilde{S}_{m}-\nu\right)^{2}}{K}\right) \\
& =E\left(\frac{m \omega^{\prime} \bar{S}_{m}^{2}}{K}\left(\left(\frac{m+1}{m}\right)^{2} \bar{S}_{m}^{2}+\nu^{2}-2 \nu\left(\frac{m+1}{m}\right) \bar{S}_{m}\right)\right) .
\end{aligned}
$$


Since $\left(\frac{m+1}{\nu}\right) \bar{S}_{m} \sim \operatorname{Gamma}(m, 1)$, thus

$$
J_{1} \leq \frac{\nu^{4} \omega^{\prime}(m+6)}{K(m+1)}
$$

Now, since $\tilde{S}_{N_{m}-m} \sim \operatorname{Gamma}\left(\left(N_{m}-m\right), \frac{\nu}{\left(N_{m}-m\right)}\right)$,

$$
\begin{aligned}
J_{2} & =\nu^{2} E\left(\frac{N_{m}-m}{\left(N_{m}+1\right)^{2}}\right) \leq \nu^{2} E\left(\frac{N_{m}-m}{m\left(N_{m}+1\right)}\right) \\
& \leq \nu^{2} E\left(\left(\frac{N_{m}}{m}-1\right) \frac{1}{\left(N_{m}+1\right)} I\left(N_{m}>m\right)\right) \\
& \leq \nu^{2} E\left(\frac{1}{\left(N_{m}+1\right)}\right) \\
& \leq \frac{\nu^{4} \omega^{\prime} m}{K(m+1)} .
\end{aligned}
$$

Similarly we can obtain

$$
J_{3} \leq \frac{\nu^{4} \omega^{\prime}}{K(m+1)}
$$

Substituting A.8, A.9 and A.10 in A.6 and using $R\left(\widehat{\gamma}_{N_{m}}, \gamma\right) \leq \omega 6^{\prime}$, it is sufficient that

$$
K=\frac{A^{\prime}\left(\theta g^{\theta-1}(t) g^{\prime}(t)\right)^{2}(2 m+7)}{m+1} .
$$

Proof of Theorem 3.2.

$$
\begin{aligned}
R\left(\widehat{\gamma}_{N_{m}}, \gamma\right) & =A^{\prime} E\left(\left(\widehat{\gamma}_{N_{m}}-\gamma\right)^{2}\right) \\
& \approx \frac{A^{\prime}\left(\theta g^{\theta-1}(t) g^{\prime}(t)\right)^{2}}{\nu^{4}}\left[E\left(\widehat{\nu}_{N_{m}}^{2}\right)+\nu^{2}-2 \nu E\left(\widehat{\nu}_{N_{m}}\right)\right]
\end{aligned}
$$

where we derive expressions for $E\left(\widehat{\nu}_{N_{m}}\right)$ and $E\left(\widehat{\nu}_{N_{m}}^{2}\right)$ using the same techniques as adopted by Mahmoudi and Roughani (2015), Roughani and Mahmoudi (2015) and Mahmoudi and Lalehzari (2017). On similar lines we can also derive the results of Theorems 3.3 and 3.4 .

\section{References}

[1] Ahsanullah, M. (1980). Linear prediction of record values for the two parameter exponential distribution. Annals of the Institute of Statistical Mathematics 32:A 363-368. 
[2] Burr, I. W. (1942). Cumulative frequency functions. The Annals of Mathematical Statistics 13: 215-232.

[3] Chaturvedi, A. and Alam, Md. W. (2010). UMVUE and MLE in a family of lifetime distributions. Journal of the Indian Statistical Association 48:2 189-213.

[4] Chen, Z. (2000). A new two- parameter lifetime distribution with bathtub shape or increasing failure rate function. Statistics $\&$ Probability Letters 49: 155-161.

[5] Cislak, P. J. and Burr, I. W. (1968). On a general system of distributions; I. Its curve-shape characteristics; II. The sample median. Journal of the American Statistical Association 63: 627-635.

[6] Davis, D. J. (1952). The analysis of some failure data. Journal of the American Statistical Association 47: 113-150.

[7] Gurvich, A. T., Dibenedetto, A. T. and Rande, S. V. (1997). A new statistical distribution for characterizing the random strength of brittle materials. Journal of Materials Sciences 32: 2559-2564.

[8] Khan, R. U. and Zia, B. (2009). Recurrence Relations for single and Product moments of record values from Gompertz distribution and a characterization. World Applied Sciences Journal 7:10 1331-1334.

[9] Johnson, N. L. and Kotz, S. (1970). Continuous Univariate Distributions-I. John Wiley and Sons, New York.

[10] Isogai, E. and Uno, C. (1994). Sequential Estimation of a Parameter of an Exponential Distribution, Annals of Institute of Statistical Mathematics 46: 77-82.

[11] Kleiber, C. (2004). Lorenz ordering of order statistics from log-logistic and related distributions. Journal of Statistical Planning and Inference 120: $13-19$.

[12] Lai, C. D., Xie, M. and Murthy, D. N. P. (2003). Modified Weibull model. IEEE Transactions Reliability 52: 33-37.

[13] Ljubo, M. (1965). Curves and concentration indices for certain generalized Pareto distributions. Statistical Review 15: 257-260. 
[14] Lomax, K. S. (1954). Business failures. Another example of the analysis of failure data. Journal of the American Statistical Association 49: 847852.

[15] Mahmoud, M. A. W. and Al-Nagar, H. SH. (2009). On generalized order statistics from linear exponential distribution and its characterization. Statistical Papers 50: 407-418.

[16] Mahmoudi, E. and Lalehzari, R. (2017). Bounded risk estimation of the hazard rate functionof the exponential distribution: Two-stage procedure. Sequencial Analysis 36:1 38-54.

[17] Mahmoudi, E. and Roughani, G. (2015). Bounded Risk Estimation of the Scale Parameter of a Gamma Distribution in a Two-Stage Sampling Procedure. Sequencial Analysis 34:1 25-38.

[18] Moore, A. H. and Bilikam, J. E. (1978). Bayesian estimation of parameters of life distributions and reliability from type II censored samples. IEEE Transactions Reliability R:27 64â€"67.

[19] Mukhopadhyay, N. (1980). A Consistent and Asymptotically Efficient Two-Stage Procedure to Construct Fixed-Width Confidence Interval for the Mean, Metrika 27: 281-284.

[20] Mukhopadhyay, N. (1994). Improved Sequential Estimation of Means of Exponential distributions, Annals of Institute of Statistical Mathematics 46: $509-519$.

[21] Mukhopadhyay, N. and Datta, S. (1995). On Fine-Tuned Bounded Risk Sequential Point Estimation of the Mean of an Exponential Distribution, South African Statistical Journal 29: 9-27.

[22] Mukhopadhyay, N. and Pepe, W. (2006). Exact Bounded Risk Estimation When the Terminal Sample Size and Estimator Are Dependent: The Exponential Case, Sequential Analysis 25: 85-101.

[23] Nakagami, M. (1960). The m-distribution- a general formulation of intensity distribution of rapid fading. In: Hoffman WC (ed) Statistical Method in Radio Wave Propagation. Pergamon, Oxford 3-36.

[24] Nikulin, M. and Haghighi, F. (2006). A chi-squared test for the generalized power weibull family for thehead-and-neck cancer censored data. Journal of Mathematical Sciences 133:3 1333- 1341. 
[25] Patel, J. K., Kapadia, C. H. and Owen, D. B. (1976). Handbook of Statistical Distributions. Marcel Dekker, New York.

[26] Rohtagi, V. K. and Saleh, A. K. Md. E. (2012). An Introduction to Probability and Statistics, Second Edition. John Wiley 85 Sons, U.K.

[27] Roughani, G. and Mahmoudi, E. (2015). Exact Risk Evaluation of the Two-Stage Estimation of the Gamma Scale Parameter Under Bounded Risk Constraint. Sequencial Analysis 34:3, 387-405

[28] Sinha, S. K. (1986). Reliability and Life Testing. Wiley Eastern Limited, New Delhi.

[29] Starr, N. and Woodroofe, M. (1972). Further Remarks on Sequential Estimation: The Exponential Case, The Annals of Mathematical Statistics 43: 1147-1154.

[30] Stein, C. (1945). A Two Sample Test for a Linear Hypothesis Whose Power Is Independent of the Variance, The Annals of Mathematical Statistics 16: 243-258.

[31] Stein, C. (1949). Some Problems in Sequential Estimation (abstract), Econometrica 17: 77-78.

[32] Stoner, E. G., Edie, D. D. and Durham, S. D. (1994). An end-effect model for the single-filament tensile test. Journal of Materials Sciences 29: 6561-6574.

[33] Tadikamalla, P. R. (1980). A look at the Burr and related distributions. International Statistical Review 48: 337-344.

[34] Takada, Y. (1986). Non-Existence of Fixed Sample Size Procedures for Scale Families, Sequential Analysis 5: 93-101.

[35] Takada, Y. and Nagata, Y. (1992). Fixed-Width Sequential Confidence Interval for the Mean of a Gamma Distribution, Journal of Statistical Planning and Inference 44: 277-289.

[36] Tyagi, R. K. and Bhattacharya, S. K. (1989). A note on the MVU estimation of reliability for the Maxwell failure distribution. Estadistica 41: 73-79.

[37] Uno, C., Isogai, E., and Lim, D. L. (2004). Sequential Point Estimation of a Function of the Exponential Scale Parameter, Austrian Journal of Statistics 33: 281-291. 
[38] Voda, V. G. (1978). Concerning an application of the Weibull distribution to reliability aspects in metrology. Metrologia Aplicată 25: 123- 124 .

[39] Woodroofe, M. (1977). Second-Order Approximations for Sequential Point and Interval Estimation, Annals of Statistics 5: 984-995.

[40] Xie, M. and Lai, C. D. (1995). Reliability analysis using an additive Weibull model with bathtub- shaped failure rate function. Reliability Engineering and System Safety 52: 87-93.

[41] Xie, M., Tang, Y. and Goh, T. N. (2002). A modified Weibull extension with bathtub-shaped failure rate function. Reliability Engineering and System Safety 76: 279-285.

[42] Zacks, S. (2009). The Exact Distributions of the Stopping Times and Their Functionals in Two-Stage and Sequential Fixed-Width Confidence Intervals of the Exponential Parameter, Sequential Analysis 28: 69-81.

[43] Zacks, S. and Khan, A. (2011). Two-Stage and Sequential Estimation of the Scale Parameter of a Gamma Distribution with Fixed-Width Intervals, Sequential Analysis 30: 297-307.

[44] Zacks, S. and Mukhopadhyay, N. (2006a). Bounded Risk Estimation of the Exponential Parameter in a Two-Stage Sampling, Sequential Analysis 25: 437-452.

[45] Zacks, S. and Mukhopadhyay, N. (2006b). Exact Risks of Sequential Point Estimators of the Exponential Parameter, Sequential Analysis 25: 203-220. 